\title{
Jürgen Zinnecker
}

\section{„Das Problem der Generationen“ Überlegungen zu Karl Mannheims kanonischem Text}

\section{Zur Einführung}

Für die sozialwissenschaftliche und historische Debatte im deutschsprachigen Raum ist kennzeichnend, daß sie sich ausdrücklich oder implizit auf ein Konzept bezieht, das in den 20er Jahren des vergangenen Jahrhunderts entwickelt worden ist und das sich mit der Person von Karl Mannheim verbindet. Sein grundlegender Aufsatz "Das Problem der Generationen“ von 1928 dient der cinschlägigen deutschen und internationalen Fachliteratur während der gesamten zweiten Hälfte des 20. Jahrhunderts bis hinein in die Gegenwart als kanonischer Referenztext. Kanonische Referenz bedeutet, daß Mannheims Konzeption, oder Teile davon, in der Mehrzahl der Fälle als relativ oberflächliche Legitimation für die eigenen Untersuchungen herhalten mußte. Nur in ganz wenigen Ausnahmefälle versuchten Autoren, den Ansatz von Mannheim kritisch aufzugreifen und weiterzuentwickeln ${ }^{1}$. Es erscheint daher dringend geraten, sich der kunstvoll gefügten Theorie-Architektur zuzuwenden, die dem klassisch gewordenen Referenztext zugrunde liegt. Das soll in Teil II dieses Aufsatzes geschehen. In Teil III wird dann auf die Frage nach der Aktualität des Textes hin fokussiert. In welchem Verhältnis steht die begriffliche Fassung, die Mannheim dem "Problem der Generationen“ gegeben hat, stehen die Fragestellungen, die er vor fast einem Jahrhundert zu bearbeiten suchte, zu den heutigen Fragestellungen und zum aktuellen theoretischen Bedarf? Aus dieser Befragung werden abschließend einige Anregungen für eine künftige Revision der in dem klassischen Text vorgegebenen Rahmung des Generationskonzeptes abgeleitet.

1 Vgl. hierzu den Absatz "Zur wissenschaftlichen Rezeption in der zweiten Jahrhunderthälfte" weiter unten. 


\section{Karl Mannheim historisch. Eine Interpretation des theoretischen Ankertextes}

Die Analyse von (Jugend-)Generationen verankert sich bis heute an einem grundlegenden Text, der aus dem ersten Drittel des 20. Jahrhunderts stammt. Er stamme aus der Feder von Karl Mannheim, dem Heidelberger, später Frankfurter Philosophen und Soziologen. Er faßte 1928 „Das Problem der Generationen“ in eindrucksvoller Weise zusammen, so wie es sich ihm im ersten Quartal des Jahrhunderts darstellte. Zu Recht gilt diese Studie seither unter Sozialwissenschaftlern und Historikern als erste Referenz und als Basis für eigene Untersuchungen, wie gleich zu zeigen sein wird. Gleichwohl ist der grundlegende Aufsatz mittlerweile, ein Dreivierteljahrhundert nach seiner Erstpublikation, selbst Geschichte, und wir können ihn und seinen Autoren als Zeitzeugen befragen. Welche Fragen wurden im ersten Terzil des alten Jahrhunderts an das Deutungsmuster Generation gestellt, welche Antworten wurden versucht, und wie verhalten sich damalige Fragen und Antworten zur Debatte um das Deutungsmuster Generation zu Beginn des neuen Jahrhunderts? Ich gebe einige knappe Hinweise zum historischen Kontext der Studie, ehe ich versuche, die Architektur dieser theoretischen Skizze zu interpretieren.

\section{Der historische Kontext}

Fachliche Diskurse der 1920er Jahre

Vorab ist festzuhalten, daß Mannheim mit seiner Schrift nicht eine neue wissenschaftliche Debatte eröffnete, sondern daß er mit ihr ein Jahrzehnt intensiver Debatte um die Frage der Generationen zusammenfaßte und abschloß. Leitend in diesem Diskurs waren, wenigstens in Deutschland, Kultur- oder Geisteswissenschaftler - Historiker, Philosophen, Kunst- und Literaturwissenschaftler. Mannheim sicherte der noch jungen Disziplin Soziologie eine Stimme in diesem wissenschaftlichen Dialog. Er verfaßte den Aufsatz aufgrund einer umfassenden Sichtung insbesondere der französischen und deutschen Literatur der davor liegenden Jahre. Bei der Rezeption der französischen Tradition und ihrer Geschichte konnte Mannheim sich auf ein einige Jahre zuvor erschienenes umfangreiches Standardwerk stützen (F. Mentré 1920). Was die deutsche historisch-qualitative Tradition anlangt, so verweist er wiederholt und nachdrücklich auf Wilhelm Dilthey (geb. 1833), den er als den Vater des historischen Generationenbegriffs ansah und dessen teilweise vergriffenen Publikationen seit Beginn der 1920er Jahre im Rahmen einer Gesamtausgabe des Werkes wieder aufgelegt und neu zugänglich wurden. Dazu zählen insbesondere 1922 das „Leben Schleiermachers“ (ursprünglich 1867) und 1924 „Über das Studium der Geschichte der Wissenschaften vom Menschen, der Gesellschaft und dem Staat“ (ursprünglich 1875)². Für die bedeutungs-

2 W. Dilthey (1927/2001, S. 217) bemerkt zur Genese des Begriffs Generation in seiner Werk- 
volle Verknüpfung von Generation und Jugendentwicklung beruft Mannheim sich auf den in der Nachfolge Diltheys stehenden Eduard Spranger (geb. 1882) und dessen in jenen Jahren aktuelles Standardwerk über die „Psychologie des Jugendalters" (1925).

Die Thematik der historischen Generationen war in den 1920er Jahren zunächst anhand der kulturellen Generationen am Beispiel von Schriftstellern und Künstlern entwickelt worden. Mannheim beruft sich hier vor allem auf den Literaturwissenschaftler und Germanisten Julius Petersen (1926) und auf den Kunsthistoriker Wilhelm Pinder (1926). Eine philosophische Fundierung erhielt das Problem der Generationen durch Martin Heidegger (1927), dessen vieldiskutierte aktuelle Neuerscheinung über "Sein und Zeit" Mannheim dazu anregte, historische Generationen unter der Perspektive von Schicksalsgemeinschaften zu sehen ${ }^{3}$. Wie man erkennen kann, verarbeitete Mannheim unterschiedliche geisteswissenschaftliche Studien, die gerade aktuell, d.h. in den 1920er Jahren erschienen waren und die seinerzeit (und z.T. auch noch heute) hohes Ansehen genossen, zu seiner politisch-gesellschaftlichen Konzeption von historischen (Jugend-)Generationen. Die Beschränkung der Literatur auf zwei kulturelle Räume, den französischen und den deutschsprachigen, spielt in der Geschichtsphilosophie und Wissenssoziologie des jungen Mannheim insgesamt eine bedeutsame Rolle (vgl. Mannheim 1925/1984). Er verband damit eine dichotome Typenlehre des rationalen, aufklärerischen, und des konservativen, romantischen Weltbildes. Die rationale Seite ordnete er der französischen Geistesgeschichte zu, die romantische der deutschen. Innerhalb der Gesellschaftslehre sieht er die Empirie (Positivismus) in Frankreich, die geisteswissenschaftliche Hermeneutik in Deutschland verankert. Seine Theorie der (Jugend-)Generationen soll eine Synthese dieser zwei entgegengesetzten Denk- und Analyserichtungen bilden (s.u.).

\section{Zur generationalen Lagerung des Autors}

Wie sind der Autor und sein Aufsatz biographisch-generationell zu verorten (vgl. Wolff 1978)? „Károly“ Mannheim, geboren 1893 im Budapest der österreichischungarischen Monarchie, kam 1919, nach der gescheiterten Revolution in Ungarn, als jüdischer Emigrant ins Deutsche Reich. In Budapest hatte er, als junger Privatgelehrter nach der Promotion, einem Kreis um Georg Lukács angehört. Dirk Käsler (1984, S. $471 \mathrm{ff}$.) rechnet ihn in seiner Geschichte der frühen deutschen Soziologie, neben Theodor Geiger oder Max Horkheimer, zur "Generation der Urenkel ${ }^{\star}$ des Faches, die durch das Großereignis des Ersten Weltkrieges maßgeblich

geschichte: „Ich habe zuerst 1865 im Aufsatz über Novalis den historischen Begriff der Generationen angegeben und benutzt, dann in größerem Umfang in Schleiermacher Bd. I verwertet und dann 1875 in dem Aufsatz über das Studium der Geschichte der Wissenschaften vom Staat usw. den historischen Begriff der Generation und mit ihm zusammengehörige Begriffe entwickelt."

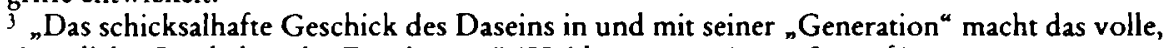
eigentliche Geschehen des Daseins aus" (Heidegger 1927/1953, S. 384 f.). 
geprägt worden waren. Käsler hebt den Bruch mit der bildungsbürgerlichen Welt des 19. Jahrhunderts in dieser Soziologen-Generation und deren Suche nach "neuer Sachlichkeit “ hervor. Für den jungen Mannheim kamen zwei besondere biographische Motive hinzu. Er war auf der Suche nach einer nicht-jüdischen Identität, und er wollte die frühe Begegnung mit dem parteilichen theoretischen Marxismus ${ }^{4}$ intellektuell bearbeiten. Er fand bekanntlich eine Lösung in der Selbst-Identifikation mit der Idee des im sozialen Raum frei schwebenden, nicht durch soziale Gruppeninteressen gebundenen Wissenschaftlers. In Abgrenzung von der marxistischen Ideologienlehre entwickelte Mannheim in den 1920er Jahren eine Wissenssoziologie, die die Seinsgebundenheit aller Ideen - nicht nur der interessengebundenen, ideologisch verzerrten - anerkannte, diese existentielle Bindung aber nicht nur auf die soziale Klassenlage beschränkt sehen wollte. In diesem Zusammenhang gewann für ihn die Bindung des Denkens an die Lagerung von historischen Generationen eine besondere strategische Bedeutung. Mit einer wissenssoziologischen Arbeit über das deutsche konservative Denken habilitierte er sich 1926 in Heidelberg, nicht ohne juristische Anfechtung aufgrund seiner ungarischen Staatsangehörigkeit ${ }^{5}$. Für kurze Zeit hatte er einen Soziologie-Lehrstuhl an der neu gegründeten Frankfurter Universität inne. Bereits 1933, nach seiner Entlassung aus dem Beamtenverhältnis, mußte Mannheim zum zweiten Mal emigrieren. Seine dritte Biographie und akademische Laufbahn begann und beendete er in London.

\section{Zur wissenschaftlichen Rezeption in der zweiten Jabrhunderthälfte}

Die Basisschrift von Mannheim erfuhr erst nach dem Zweiten Weltkrieg eine internationale Rezeption, insbesondere im angelsächsischen ${ }^{6}$ und im deutschen Raum. In Deutschland wurde "Das Problem der Generationen" wiederholt an prominenter Stelle nachgedruckt. So wurde der Text in den verbreiteten Sammelband der Schriften zur Wissenssoziologie aufgenommen, der Mannheim in der westdeutschen Soziologie wieder bekannt machte (Mannheim 1964). Ludwig von Friedeburg (1970) räumte dem Aufsatz einen prominenten Platz in seinem Sammelband zur Jugendsoziologie ein, mit dem die moderne, Nach-68er Tradition der Subdisziplin eröffnet wurde. Martin Kohli nahm ihn, in gekürzter Form, in seine Textsammlung zur „Soziologie des Lebenslaufs“ (1978) auf, mit der dieses Wissenschaftsfeld im deutschen Sprachraum abgesteckt wurde. In der internationalen Rezeption wird der Text von Mannheim in den letzten Jahren etwa zur Hälfte nach der - schwer zugänglichen - Erstfassung von 1928 zitiert, während die übrigen Autoren spätere Wiederabdrucke des Textes als Referenz angeben, wie u.a. der Social Citation Index ausweist. In der Mehrheit der Fälle handelt es

4 Bedeutsam wurde für ihn die Auseinandersetzung mit Georg Lukács und insbesondere dessen Werk „Geschichte und Klassenbewußtein" (1923).

5 Die Habilitationsschrift von 1925 wurde erst 1984 vollständig veröffentlicht. Vgl. Mannheim (1984).

6 Z.B. Simirenko (1966); Demartini (1985). 
sich bei den Verweisen um eine konventionelle Referenz gegenüber dem historischen Ankertext, ohne kritische, eigenständige Position gegenüber dem Original. Auf einige bemerkenswerte Ausnahmen sei kurz hingewiesen. In der soziologischen Rezeption wurde insbesondere die Möglichkeit ausgelotet, Mannheims Konzept mit dem Kohortenansatz zu verknüpfen und damit für die nach dem Zweiten Weltkrieg in Deutschland erstarkte empirische Sozialforschung anwendbar zu machen (z.B. Pfeil 1967; Buchhofer, Friedrich, Lüdtke 1970; Breitsamer 1976; Kreutz 1983; Weymann 2000; vgl. für die anglo-amerikanische Seite N. B. Ryder 1965). Eine Minderheitenposition vertrat hier Joachim Matthes (1985), der Mannheims Theorie stärker in Richtung moderner Zeit-Soziologie - weg von ciner gruppensoziologischen Perspektive - interpretieren wollte. Diese und weitere Neuinterpretationen waren durch die posthume Veröffentlichung früher kultursoziologischer Schriften Mannheims $(1980 ; 1984)$ inspiriert. Hier läßt sich auch die Wiederbelebung des Mannheimischen Konzepts der „konjunktiven Erfahrung“ durch Ralf Bohnsack (1998) einordnen. Während der 1980er Jahre rezipierte die historische Sozial- und Sozialisationsforschung das Konzept der Generationen (z.B. Ulrich Herrmann 1987). In der ostdeutschen Jugendforschung wurde Mannheim erst mit dem Ende der alten DDR wieder rehabilitiert (Friedrich 1990).

Die Attraktivität des wissenschaftlichen Deutungsmusters, das uns Mannheim in seinem Aufsatz "Das Problem der Generationen " hinterlassen hat, beruht nicht zuletzt auf den vielen kunstvollen theoretischen Synthesen, die er dabei hergestellt hat. Ich will im weiteren Verlauf einige wesentliche Syntheseleistungen von Mannheim aufgreifen und daraufhin befragen, ob und in welcher Interpretation sie sich Anfang des 21. Jahrhunderts weiterhin als tragfähig erweisen. Dabei werde ich auf folgende Punkte eingehen: die Auffassung vom gesellschaftlichen Wandel, die Anbindung an die Jugendforschung der Zeit, die Zielgerichtetheit (Entelechie) von Generationen, die Verschweißung von Alter, Geburt und historischer Periode, die Dreistufigkeit des Generationenkonzeptes (Lagerung, Zusammenhang und Einheit der Generation) und schließlich die Integrienung von qualitativer und quantifizierender Generationenforschung.

\section{Die Dynamik des gesellschaftlichen Wandels}

„Das Generationsphänomen ist einer der grundlegenden Faktoren beim Zustandekommen der historischen Dynamik." (Mannheim 1964, S. 565) Aufgabe des Deutungsmusters Generation ist es in den Augen Mannheims, sozialen Wandel in der Geschichte zu erklären und so eine ,angewandte historische Soziologie ${ }^{\text {“ }}$ (ebd., S. 523) mitzubegründen. In jeder neuen Generation lagere eine „schlummernde Potentialität “ (ebd., S. 550), die unter bestimmten historischen Bedingungen aktiv werden könne. Allerdings seien Generationen nur einer der wirksamen Faktoren. Mannheim wendet sich gegen den „Monismus“ vieler älterer Generationentheorien, die versucht hätten, ,aus diesem einen Faktor die Gesamtdynamik im historischen Geschehen zu erklären “ (ebd., S. 555), ebenso wie er sich 
gegen den „Monismus" marxistischer Theorien ausspricht, in dem allein Klassenlage und Klassenbewußtein (Lukács) als historisch wirkmächtig anerkannt werden. Statt dessen plädiert er für eine mehrfaktorielle Theorie gesellschaftlichen Wandels?

Anders als die französische Aufklärung des 18. Jahrhunderts, in deren Theorien der Generationswechsel „als einer der wesentlichsten treibenden Faktoren im Fortschritt betrachtet wurde" (ebd., S. 515f.), rechnet Mannheim den Wechsel von Generationen nicht einem linearen Faktor Fortschritt zu. Er folgt hier eher der historisch-romantischen Linie, die Generationen mit einmaliger Neuschöpfung oder mit einem Bruch der historischen Kontinuität verbindet (vgl. Bilstein 1996). Er hebt hervor, daß die jeweiligen ,inhärierenden Tendenzen“ (Mannheim 1964, S. 518 f.), denen eine sichtbare Generationseinheit Ausdruck verleiht, sich antithetisch, polar, zu vorangegangenen historischen Tendenzen entwickelten. Das Deutungsmuster Generation gibt ihm so die Möglichkeit, historischen Wandel zu thematisieren, ohne ihn linear - zielgerichtet - denken zu müssen. Das schafft Anschlußstellen für postmoderne Theorien, die historische Entwicklung in Brüchen und Paradoxien denken ${ }^{8}$.

Mannheim verknüpft die historische Wirksamkeit des Faktors Generation auf anregende Weise mit der Geschwindigkeit bzw. der Beschleunigung des Wandels in einer Gesellschaft. Statische Gesellschaften, in denen der historische Wandel sich langsam und kontinuierlich vollziehe, seien Gesellschaften ohne ausgewiesene Generationsgestalten. Je mehr sich die Dynamik des Wandels beschleunige, um so größer werde die Chance, daß es zur Ausbildung von distinkten Generationen komme. Statt Kontinuität erzeugten neue Generationen jetzt Umbrüche, da „die Generationslagerungen differenter “ werden und „die Anschlußfähigkeit des sozialen Wissens der Generationen abnimmt " - so interpretiert Weymann (2000, S. 41) den Grundgedanken von Mannheim.

Paradox mag es erscheinen, daß im heutigen Diskurs um das Deutungsmuster Generation auch die gegenteilige Auffassung vertreten wird. Der dauerhaft beschleunigte Wandel Ende des 20. Jahrhunderts verhindere geradezu die Neuausbildung ausgewiesener Generationen (so Steiner 1997, S. 18), da die hohe Geschwindigkeit der Veränderungen eine ständige Überlagerung und Verdeckung von Generationszusammenhängen erzeuge. Läßt sich das Paradox auflösen?

7 Vgl. eine parallele Abgrenzung beim Vater der angloamerikanischen Kohortenforschung. "The new cohorts provide the opportunity for social change to occur. They do not cause change; they permit it." (Ryder 1965, p. 844)

8 Vgl. z. B. Hörisch 1997 b, S. 13 zum "Generationenumbruch“ zwischen der 68er und 89er Generation. Mannheims Konzept des generationellen Wandels gerät damit in ein gewisses Spannungsfeld zu Modernisierungstheorien, die den historisch-gesellschaftlichen Wandel im Modeil langfristiger Wellenbewegungen denken. Heinz Bude (1997) hat einen solchen Gegensatz zur Zivilisationstheorie von Norbert Elias herausgearbeitet, dem Assistenten von Mannheim während der kurzen Frankfurter Jahre. Eine mögliche Unvereinbarkeit der Modelle dürfte, das sei am Rande erwähnt, auch zu bestimmten Vorstellungen eines längerfristigen Wertewandels bestehen. Es ist jedenfalls auffallend, daß viele dieser Konzepte ohne eine Bezugnahme auf Generationen auskommen. 
Denkbar wäre, daß es sich um eine kurvilineare Beziehung zwischen sozialem Wandel und Generationsbildung handelt: Bis zu einer gewissen Geschwindigkeit begünstigt die sich beschleunigende Dynamik des historischen Wandels die Herausbildung erkennbarer Generationsgestalten, um dann, ab einer bestimmten Geschwindigkeit, eben dieses durch die Gleichzeitigkeit sich zeitlich überlappender Generationsgestalten zu verhindern.

\section{Der Anschluß an die Jugendforschung}

Die Generationstheorie von Mannheim, entwickelt zur Zeit der bündischen Jugend, steht unter dem Eindruck der historischen Jugendbewegung in Deutschland, der Erwartung einer Erneuerung durch die jüngere Generation (der Neue Mensch), einer ersten Hochphase von akademischer Jugendtheorie und Jugendforschung (vgl. den Sammelband von Koebner, Janz, Trommler 1985). Unter diesem Sternenhimmel erscheint die Generationentheorie von Mannheim, die die Jugendphase ins Zentrum stellt, mehr als plausibel. Da die Auseinandersetzung mit der Jugendphase im allgemeinen nicht ganz unabhängig vom Lebensalter der Wissenschaftler ausfällt, sei noch hinzugefügt, daß Mannheim zum Zeitpunkt der Veröffentlichung des Textes zum Problem der Generationen erst 35 Jahre alt ist ${ }^{9}$. Er steht in einer Tradition von deutschen Generationstheorien seit dem Ende des 19. Jahrhunderts, „bei denen es sich fast ausschließlich um Jugendgenerationstheorien handelte“ (Sackmann 1992, S. 211). Seine zentrale und praktisch einzige Referenz aus dem Gebiet der sich etablierenden Jugendforschung ist, wie schon erwähnt, die von Eduard Spranger (1925) im Sinne der geisteswissenschaftlichen Psychologie und der romantisch-deutschen Bewegung seinerzeit gerade vorgelegte "Psychologie des Jugendalters“.

Mannheim argumentiert glaubwürdig und überzeugend: „Erste Eindrücke“ seien entscheidend für die "Formierung des Bewußtseins" (Mannheim, S. 36). „Die ersten Eindrücke haben die Tendenz, sich als natürliches Weltbild festzusetzen “ (ebd., S. 536). Sie bestimmen die Bearbeitung der nachfolgenden Erlebnisse und Erfahrungsaufschichtungen. Die Jugendphase ist besonders für die Erfahrungen im gesellschaftlich-politischen Raum prägend, auf die es ihm ankommt. Er will ja den vorangegangenen Generationstheorien in Deutschland, die sich auf Kunst- und Literaturgeschichte (Petersen 1926; Pinder 1926 u.a.) bezogen, eine Theorie der politisch-gesellschaftlichen Generationsbildung an die Seite stellen.

\footnotetext{
9 Mannheim (1951) hat sich noch einmal, 1941, in einem fachlichen Aufsatz zum „Problem der Jugend in der modernen Gesellschaft" geäußert. Dabei geht es ihm um die Frage, wie es westlichen Demokratien wie derjenigen Großbritanniens gelingen könne, das innovative Potential ihrer Jugenden zu mobilisieren. Zwar geht es ihm auch in diesen Kriegsjahren um Fragen des gesellschaftlichen Wandels, aber ohne Bezugnahme auf eine Theorie der Generationen. Dieser Beitrag wird von Helmut Scbelsky (1957) in der "Skeptischen Generation" referiert, wobei er den älteren Artikel zum Problem der Generationen nicht erwähnt, wahrscheinlich nicht kennt - obgleich sein eigenes einleitendes Thema die Abfolge politischer Jugendgenerationen im 20. Jahrhundert in Deutschland ist.
} 


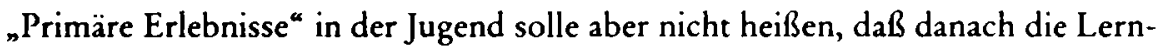
prozesse zu Ende seien. Mannheim geht, hierin folgt er dem Vordenken Wilhelm Pinders $(1926)^{10}$ und ist zugleich ganz modern, von einem lebenslaufbegleitenden politischen Lernen aus. Primär heißt nur, daß eine eigene "Dialektik“ des Lernens durch sie in Gang gesetzt werde 11. Es ist also nicht korrekt, wenn Mannheim gelegentlich vorgehalten wird, er unterstelle eine lebenslange starre inhaltliche Prägung z. B. politischer Orientierungen seit der Jugendzeit. „... die Prädominanz der ersten Eindrücke bleibt auch dann lebendig und bestimmend, wenn der ganze darauffolgende Ablauf des Lebens nichts anderes sein sollte, als ein Negieren und Abbauen des in der Jugend rezipierten ,natürlichen Weltbildes" “ (Mannheim 1964, S. 537). In solchen und ähnlichen Formulierungen erweist sich die Anschlußfähigkeit der Überlegungen von Mannheim an die moderneren Begriffe des biographischen Lernens bzw. der biographischen Sozialisation.

Gleichwohl bleibt die grundsätzliche Frage: Ist die Jugendgenerationstheorie von Mannheim als zeitgenössisches Produkt des in der deutschen Geschichte zu Anfang des Jahrhunderts besonders virulenten Mythos Jugend zu verstehen? War sie vom Siegeszug einer akademischen Jugendtheorie in jenen Jahren beeinflußt? Mit dem Verblassen des Mythos im Verlauf des 20. Jahrhunderts sinkt auch die Überzeugungskraft einer Generationstheorie, die sich stark auf die Jugendphase bezieht. Mittlerweile ist die Jugendphase in mehrfacher Hinsicht in eine kritische Schieflage geraten, was ihren Status, ihr Ansehen in der Gesellschaft betrifft. Whir können von einer „Entzauberung “ der jüngeren Generation sprechen. Das Problem der gewandelten Jugendphase soll weiter unten aufgegriffen werden.

\section{Die Entelechie von Generationen}

Im Anschluß an Pinder (1926), der ein Theorem der modernen Kunstgeschichte der Zeit auf künstlerische Generationen überträgt, nimmt Mannheim auch für politisch-gesellschaftliche Generationen ein "formendes Prinzip“, einen „einheitlichen, treibenden Impuls“ an, der mit dem philosophischen Begriff der „Entelechie" belegt wird ${ }^{12}$. Entelechie einer Generation ist nach ihm [W. Pinder] Ausdruck der Einheit ihres ,inneren Zieles“, Ausdruck eingeborenen Lebens- und

10 Pinder (1926, S. 54f.) argumentiert mit dem "Lebensaltersstil ganzer Generationen ${ }^{*}$, der zur "Polyphonie" einer Zeitepoche beitragen könne.

$11 \mathrm{Vgl}$. die Auslegung bei $A$. Weymann 2000, S. 41: „... die erste Stufe einer lebenslangen Erfahrungsaufschichtung. Sie bildet den Filter für alle weiteren Ereigniswahrnehmungen und Erlebnisgehalte."

12 Der philosophische Begriff „Entelechie“ wurde maßgebend für die frühe, an der endogenen Entwicklung des Organismus orientierte Entwicklungspsychologie. Entelechie bezeichnet eine Substanz, eine zielstrebige Kraft oder eine Fähigkeit, welche die weitere Entwicklung eines Organismus bewirkt bzw. reguliert.

Man könnte das Problem, das damit angesprochen wird, heute vielleicht in einer Bcgrifflichkeit formulieren, die der französische Soziologe und Anthropologe Pierre Bourdieu vorgeschlagen hat, nämlich als "Habitus“, der in diesem Fall aus einer generationellen Lagerung hervorgeht. 
Weltgefühls (Mannheim 1964, S. 518). Die ,inhärierende Tendenz “ geht aus der generationellen Lagerung hervor, bezeichnet die zeitbezogenen Grundprobleme nicht die unterschiedlich ausfallenden Lösungen -, vor die sich eine Generation in ihren prägenden Jahren jeweils gestellt sieht.

Unter welchen Bedingungen tritt die "inhärierende Tendenz" hervor, wird sie wirksam, und wann bleibt sie latent? Hierzu finden wir im Text viele ausgearbeitete Überlegungen von Mannheim, die er im Anschluß an die zeitgenössischen Generationstheorien präsentiert. So unterscheidet er beispielsweise in Anlehnung an den französischen Theoretiker Mentré (1920) „feste ${ }^{*}$ von eher flüchtigen ("flüssigen") Sozialformen. Ebenfalls in Anlehnung an Mentré leitet er daraus die These ab: „... die Generationsrhythmik scheint eher in den "séries“, also in der Abfolge freier Gruppierungen der Menschen (Salons, literarische Gruppen usw.) wahrnehmbar zu sein, als etwa im Schoß der Institutionen, die Habitus, Aktionsweise durch Bestimmungen oder durch gemeinsame Werkleistungen im voraus weitgehend festlegen und dadurch das Neue der heranwachsenden Generationen verdecken“ (Mannheim 1964, S. 513). Übertragen wir diesen Gedanken auf die Gegenwart, so sollte sich analog prognostizieren lassen, daß wir heute die „reinste ${ }^{a}$ Ausdrucksform junger Generationen und die Ausbildung von polaren Generationseinheiten in Moden, Musikstilen, Szenen, also auf dem Gebiet der jugendkulturellen Aktivitäten antreffen. Weniger stark sollten sich Generationsgestalten dagegen im Feld des Schul- und Ausbildungssystems ausprägen.

\section{Die Ausdifferenzierung von Lage, Zusammenhang und Einheit in einer Generation}

Zentral für das Modell der Generationen bei Mannheim ist die begriffliche Trias "Generationslagerung, Generationszusammenhang, Generationseinheit" (ebd., S. $541 \mathrm{ff}$.). Er entwickelt diese Dreieinheit in Analogie zur sozialen Klassenlage, wobei er Generationen als einen „besonderen Typus der sozialen Lagerung “ (ebd., S. 528) verstanden wissen will. Durch den zeitlich umgrenzten Zeitraum, in den alle eingebunden sind, erwächst eine historische Potenz - die durchaus latent und unrealisiert bleiben kann -, nämlich „eine potentielle Partizipation an gemeinsam verbindenden Ereignissen und Lebensgehalten" (ebd., S. 536). Erst wenn die Individuen, die sich in derselben Generationslagerung befinden, tatsächlich auch „am gemeinsamen Schicksal“ „partizipieren“, will Mannheim von einem Generationszusammenhang sprechen (ebd., S. 547).

Wenn sich so ein historischer Generationszusammenhang hergestellt hat, können sich auf dieser Basis noch kleinere Generationseinbeiten herausbilden. Als Kriterium solcher Einheiten führt Mannheim an, es handele sich um n... ein einheitliches Reagieren, ein im verwandten Sinne geformtes Mitschwingen und Gestalten ..." (ebd., S. 547). Erst auf dieser dritten Stufe seien konkrete Gruppenbildungen von Menschen zu erwarten, die sich auch persönlich kennen und soziale Netzwerke bilden, insbesondere in der Anfangsphase der Herausbildung solcher Einheiten. Generationelle Einheiten bilden oftmals ein systemisches Geflecht, das 
von Ablehnung und Konkurrenz mitbestimmt ist. Hier gelingt ihm eine weitere theoretische Synthese, indem er, in guter systemischer Manier, „polar sich bekämpfende Generationseinheiten“ in den Mittelpunkt seiner Analyse stellt. „Sie werden gerade dadurch, daß sie aufeinander, wenn auch kämpfend, abgestimmt sind, einen ,Zusammenhang 'bilden." (ebd., S. 547) Als illustrierendes Beispiel aus der Zeit der Studentenbewegung von 1968 könnte man beispielsweise die polaren Gruppen der „antiautoritären“ und der „autoritären“ (Kader) Generations-Einheiten anführen. Möglich wäre zu einer gewissen Phase auch die politische Polarisierung der Einheiten in „SDS“ und „RCDS ${ }^{\star}$. Mannheim seinerseits unterstellt als zentrale Dimension der Polarisierung in seinen historischen Beispielen auch hier wiederum einen "rationalistischen " (Aufklärung) und einen "romantischen" (Konservativismus) Pol der Generationseinheit.

\section{Die Konfigurierung von Alter, Geburt und Periode}

Bekanntermaßen, und das ist natürlich auch Mannheim und seinem Gewährsmann Pinder bewußt, ist das Deutungsmuster Generation mehrfach dimensioniert, was im Alltagsdiskurs die Möglichkeit eröffnet, mit der Semantik zu jonglieren und rhetorisch zu verblüffen (vgl. Lange 1999). Selbst wenn wir die Geschlechterfolge in der Familie, die generativen Generationen, einmal ausklammern, bleiben noch drei unterschiedliche, gebräuchliche Bedeutungen des Begriffs erhalten.

Eine erste Bedeutung kreist um die Geburtsjahre. Zur gleichen Generation zählen die Personen, die im selben Jahr oder in einander benachbarten Jahren geboren sind. Empirisch-technisch wird von Kohorten gesprochen. Wir können sie Geburts-Generationen (z.B. die 1939 Geborenen oder die nach dem Weltkrieg Geborenen) nennen.

In einer zweite Semantik geht es um alle Personen(gruppen), die zu einem bestimmten Zeitraum zeitgleich leben, unabhängig vom jeweiligen Geburtsjahr, und die an gleichen historischen Ereignissen teilnehmen. In diesem Sinn sprechen wir von zeitgeschichtlichen Generationen (z.B. eine Kriegsgeneration; die Generation der 60er Jahre).

Die dritte Bedeutung schließlich ergibt sich aus dem Lebenslauf und seiner Einteilung in distinkte Altersgruppen. Wir vergleichen die Generation der Erwachsenen mit der der Kinder, Jugendlichen, Senioren. Oftmals wird das vereinfachend und in polarisierender Absicht als Gegensatzpaar von jüngerer und älterer Generation in den Blick genommen. Wir können diesen Sprachgebrauch mit Lebensalter-Generationen umreißen.

Die heutige empirische Forschung zu historischen Generationen sieht in dieser Vielfalt des Generationenbegriffs ein entscheidendes Forschungsproblem, da hier unterschiedliche Bedeutungen und mögliche Wirkungen einer Generationszugehörigkeit unentwirrbar miteinander vermischt werden (konfundieren). Für Zwecke der Empirie wird daher versucht, diese Faktoren in unabhängig voneinander meßbare Komponenten zu zerlegen: Kohorteneffekte (Einfluß des Ge- 
burtsjahres), Periodeneffekte (Einfluß der Zeit-Ereignisse) und Alterseffekte (Einfluß des Lebensalters) werden so in ihrer Bedeutung separat analysierbar.

Wie ging Mannheim mit diesem Problem um? Er wählte eine bestimmte theoretische Konstruktion, um dem Problem der Vermischung verschiedener generationeller Komponenten zu entgehen. Er koppelte die drei Komponenten Lebensalter, Geburtsjahr und historisches Ereignis mittels starker inhaltlich-theoretischer Vorannahmen aneinander. Zunächst reduzierte er die prägenden Erlebnisse, die Menschen im Verlauf ihres Lebens begegnen können, auf eine überschaubare Lebensphase, die Jugendzeit, und begründete diese Einschränkung, die zunächst als wenig wahrscheinlicher Sonderfall angesehen werden könnte, mit plausiblen Annahmen von Entwicklungspsychologie und Jugendforschung seiner Zeit. Mit der so generierten festen Verknüpfung von Lebensphase (z.B. Pubertät um das 14. Lebensjahr) und prägendem Ereignis (z.B. Beginn des Weltkrieges 1914) läßt sich der dritte Faktor, das Geburtsjahr, gleichfalls konstant halten bzw. errechnen (1900).

Das von Mannheim entworfene Instrumentarium läßt sich in der Rhetorik wissenschaftlichen Argumentierens flexibel handhaben und wird deshalb gern genutzt. Beispielsweise kann man, mit einer gewissen Willkür, je nach Bedarf die Geburtsjahre (Kohortenzugehörigkeit) oder die Teilnahme am prägenden historischen Ereignis (Zeitzeugenschaft) in den Vordergrund der Darstellung rücken. Was jeweils Thema, was Hintergrund der Deutung ist, kann wechseln. So läßt sich im Fall der Studentenbewegung das Faktum der Teilnahme an einem zeitgeschichtlichen Ereignis (1968 an der FU Berlin) thematisieren, wobei die Frage des Jahrgangs oder des Lebensalters der Teilnehmer an dieser Bewegung zum Zeitpunkt 1968 im Hintergrund verbleibt. Oder es werden die Geburtsjahre der Studentenbewegten (Jahrgänge 1939 bis 1949) in den Vordergrund gestellt und die Jahre der prägenden Ereignisse (die Studentenjahre) bzw. das Lebensalter dabei aut diesem Weg zum variablen Lebenshorizont dieser Geburtskohorten abgewertet ${ }^{13}$.

Mannheim hat, so könnte man zusammenfassen, ein flexibles Instrument der Interpretation geschaffen, das die Synergieeffekte dreier Modi der Generationszugehörigkeit - Alter, Geburtsjahrgang, Ereignisjahr - zusammenzuführen erlaubt, das aber auch für Unschärfen und Manipulationen aller Art offen ist. Ich nenne stichwortartig einige der möglichen methodischen Zweifel an seinem Modell. Wird das Drei-Komponenten-Modell von Mannheim nicht dadurch (unzulässig) vereinfacht, daß entweder die Geburtskohorte oder die Teilnahme an einem historischen Ereignis einseitig thematisiert und die anderen Komponenten zum ungeklärten (unkontrollierten) Hintergrund der Deutung gemacht werden? Ohnehin

${ }^{13}$ Es läßt sich in den sozialwissenschaftlichen Disziplinen eine gewisse Tendenz erkennen, eine Annäherung an den Kohortenansatz zu versuchen und die Geburtsjahre in der Forschung voranzustellen. Das gilt in jedem Fall für die kohortenorientierte empirische Sozialforschung. Im Fall der historisch-sozialgeschichtlichen Generationenforschung wird dagegen oftmals die gemeinsame Teilnahme am historischen Ereignis in den Mittelpunkt der Analyse gerückt (z.B. „Als die Elektrizität kam ${ }^{\alpha}$; Das Jahr $1945^{\alpha}$ bzw. „Kriegsende“). 
läßt die Frage, welches die Lebensjahre seien, die als "Jugendphase" zu kennzeichnen seien, erhebliche Spielräume offen (Pubertät; Jugendphase; 12-14; 14-18, 19-29 Jahre usw.). Durch das Zusammenwirken der drei verbundenen Teilfaktoren suggeriert das Modell der Generationenanalyse bei Mannheim einen - empirisch erst nachzuweisenden - Synergieeffekt der Komponenten, der sich an historisch „erfolgreichen“ Generationen-Einheiten ausrichtet. Das Modell läßt zudem methodisch-skeptische Fragen wie die ins Leere laufen, welcher Aspekt der gebündelten Generationskomponenten, Alter, Geburt oder Periodenereignis, denn maßgeblich an der Wirksamkeit einer Generation beteiligt sei. Läßt sich ferner das Jugendalter generell als prägende Phase im Lebenslauf verstehen? In welchem generationellen Lebensbereich trifft die Annahme möglicherweise zu, in welchem weniger (z. B. in Politik, Ökonomie, Konsum usw.)? Welche Rolle spielt ferner die Qualität der periodischen Ereignisse (z.B. Kriegsteilnahme contra Jeans-Jugend) als konstitutives Element für eine Generation?

\section{Die Versöbnung „positivistischer" und „romantischer" Tradition}

Als letzten Gesichtspunkt der Theorie-Architektur möchte ich den Versuch von Mannheim anführen, messende und interpretierende Verfahrensweisen in der Generationen-Forschung $\mathrm{zu}$ vereinen - um nicht zu sagen $\mathrm{zu}$ „versöhnen“. Er brachte in seinem Aufsatz die "positivistische“ (Mannheim 1964, S. 509) Fassung des Generationsproblems, die er der französischen Tradition zurechnete, und die „romantisch-historische“ (ebd., S. $514 \mathrm{ff}$.) Version, nach Mannheims Auffassung der deutsche Weg, in einen wechselseitigen Dialog miteinander. Während sich die einen von der Meßbarkeit, der Chronologie des biologisch fundierten Generationswechsels fasziniert zeigten, setzten die anderen auf die "innere Erlebniszeit“ der Geisteswissenschaftler Dilthey (1922; 1927), der Germanist Petersen (1926) und der Kunsthistoriker Pinder (1926) sind ihm Kronzeugen für diese Qualität. Statt Linearität in der Abfolge der Generationen in der äußeren Zeit geht es hier um die Einmaligkeit des inneren, gleichförmig gestimmten Lebens- und Erlebnisraumes jeder Generation. Mannheim wollte in seinem Modell von Generationen beide Komponenten zu ihrem Recht kommen lassen. Eine solche Haltung korrespondierte mit seinem pragmatisch-ausgleichenden Interesse, die (bereits) in der frühen Soziologie auseinanderstrebenden Richtungen, insbesondere die messende

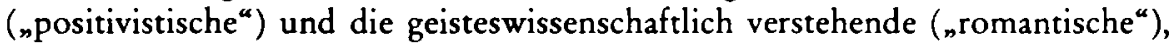
mit ihren jeweiligen Stärken bzw. Schwächen zum Tragen kommen zu lassen. Alles in allem zeigten die Fachvertreter bis heute allerdings wenig Neigung, diesem Vorschlag zu folgen. Die Rezeption des Ursprungstextes von Mannheim belegt eher die Tendenz, sich entweder auf die eine oder auf die andere Forschungsseite - Kohortenansatz oder Generationsansatz - zu schlagen und das synthetisierende Interesse des Autors zu ignorieren ${ }^{14}$.

14 Im letzten Jahrzehnt etablierte sich - zumeist unter der Flagge einer methodischen „Triangulation" - eine Forschungsbewegung in den Sozialwissenschaften, die mit der Absicht von 


\section{Karl Mannheim aktuell. Kontinuität und notwendige Revision}

Der klassische Text von Mannheim ist mittlerweile auch ein historischer Text geworden. Rund 75 Jahre Geschichte gesellschaftlicher Modernisierung und Modernisierungskrisen trennen uns von ihm. Es ist daher geboten, darüber nachzudenken, ob die leitenden Fragen, von denen er seinerzeit ausging, und die theoretischen Synthesen, die er entwickelte, für die gesellschaftliche Situation Anfang des 21. Jahrhunderts noch tragen.

\section{Thesen zur notwendigen Erweiterung der Generationentheorie}

Ich will im weiteren einige Grundannahmen von Mannheim herausstellen, die seinem theoretischen Konzept historischer Generationen zugrunde liegen, die sich für bestimmte Problemstellungen nach wie vor als sinnvoll erweisen, die aber für manche der Fragen, die sich heute stellen, insgesamt als zu eng und zeitgebunden anzusehen sind. Thesenartig formuliert, geht es um die folgenden Vorentscheidungen in Mannheims Theoriearchitektur, die sinnvollerweise zu revidieren oder wenigstens zu befragen sind.

1. Mannheim entwickelte eine Theorie der Generationen, die sich auf das Ganze der Gesellschaft richtete, also makrogesellschaftlich konzipiert war. Gegenwärtig interessieren uns - parallel hierzu - auch Generations-Probleme, die auf mikround mesogesellschaftlicher Ebene angesiedelt sind. Gegenwärtige Diskurse handeln ebenso von familialen und pädagogischen Generationenbeziehungen (Mikroebene) wie von Generationenverhältnissen, die auf der Ebene von Organisationen (z. B. Betrieb, Kirchengemeinde) oder von regional begrenzten Milieus (z. B. Stadtteile, konfessionelle Subkulturen) angesiedelt sind. Das könnte für eine künftige Weiterentwicklung einer Theorie der Generationen heißen, daß eine Rahmung angestrebt wird, dic mikro-, meso- und gesamtgesellschaftliche Prozesse gleichermaßen umschließt. Mehr noch: Auch die Prozesse der Globalisierung von Generationenverhältnissen wäre letzten Endes mit einzubeziehen.

2. Mannheim sieht in Generationen eine Treibkraft des gesellschaftlichen Wandels. Diese Frage ist zwar aktuell geblieben, aber sie erscheint ergänzungsbedürftig. Was die gegenwärtigen wissenschaftlichen Diskurse in einer voranschreitenden Moderne, in der viele der tradierten sozialen Bindemittel ihre Wirkkraft verlieren, gleichermaßen bewegt, ist die politische und soziale Integrationsfunktion. Kann Generationen eine solche integrative Rolle in der sich individualisierenden Moderne zufallen?

3. Die Generationen-Theorie Mannheims bindet sich eng an das Konzept von Jugend-Generationen, wobei eine feste Beziehung zum Faktor des historischen Wandels durch Jugend unterstellt wird. In einer Epoche des „lebenslangen Ler-

Mannheim konform geht, qualitative und quantitative Verfahren zu Forschungseinheiten zu verknüpfen. 
nens" stellt sich natürlicherweise die Frage, ob es nicht auch für die anderen Altersphasen lebensprägende Ereignisse gibt, die als Motor für die Bildung von Generationen fungieren können - jetzt aber als Kinder-Generationen oder Erwachsenen-Generationen. Zudem ist uns Heutigen die ausschließliche und privilegierte Verknüpfung von Jugendphase und Dynamik des gesellschaftlichen Wandels zweifelhaft geworden (Zinnecker 2002). Sowohl die Frage der prägenden Jahre als auch die nach den dynamischen Generationsgruppen wäre somit als eine empirische Frage freigegeben, die die Forschung zu prüfen hätte. Die kunstvollen und suggestiven Synthesen, die Mannheim seinerzeit hergestellt hat, würden auf diese Weise allerdings zerstört.

4. In der Generationentheorie von Mannheim bleibt die Frage letztlich unentschieden, ob es sich bei (Jugend)Generationen um eine universale Erscheinung menschlicher Geschichte handelt, die naturnotwendig (anthropologisch vorgegeben) in jeder Epoche und in jeder Gesellschaft anzutreffen sei - oder ob (Jugend)Generationen als distinkte Merkmale bestimmter Epochen und Kulturen anzusehen seien. Mannheim betonte - in der Tradition der positivistischen Generationentheorien - auf der einen Seite den universalistischen Charakter von Generationen in der menschlichen Gesellschaft. Der Wechsel von Generationen wäre danach ein soziobiologischer Tatbestand, überzeitlich gültig und nicht an besondere historische Epochen und Hochphasen gebunden. Auf der anderen Seite folgte er einem qualitativ differenzierenden Begriff von Generationen, wonach es nur gelegentlich zu Hochformen einer Generation kommt, während es dazwischen historische Epochen gibt, in denen dieses nicht gelingt. Damit folgt er einem substantiellen, wertenden Konzept der Generationen, das die Anerkennung von Generationen an das Vorhandensein bestimmter kultureller Qualitätsmerkmale knüpft. Eine weitere Spezifikation unterließ er. Die doppelte Bestimmung des Problems der Generationen, als einem universalistisch gültigen und einem epochenspezifischen, blieb in gewisser Weise nebeneinander stehen.

Wenn wir die Herausbildung von Generationen historisieren, also in bestimmte historische Epochen und Kulturen verlagern, ergibt sich eine interessante Folgefrage: Macht es dann nicht Sinn, nach historischen Epochen und Kulturen zu suchen, in denen die Genese von (Jugend)Generationen besonders begünstigt wurden? Wir könnten dann, analog zur Debatte um historische Hochphasen der Milieubildung, von Hochphasen in der Bildung von (Jugend)Generationen sprechen (siehe dazu weiter unten).

5. Mannheim folgte stillschweigend einem Konzept gesellschaftlicher Eliten. Die politischen oder literarisch-künstlerischen Generationen, die er als Faktor des Wandels analysierte, waren zugleich junge Eliten, zumindest wenn es um die kleinen innovativen Gruppen der generationellen Einheiten ging. Im strengen Sinn generationsfähig waren somit nur ausgewählte Bevölkerungskreise. Diese zeichneten sich insbesondere durch einen privilegierten Zugang zur dominanten Kultur - sprich Hochkultur - einer Zeit aus. Solche sozialen und kulturellen Voraussetzungen des Generationenkonzeptes waren Mannheim so selbstverständlich, $\mathrm{da}$ er sie nicht eigens thematisierte. Mittlerweile hat die Moderne eine machtvolle 
Popularkultur hervorgebracht, welche die Mehrheit aller Bevölkerungsgruppen einschließt und welche das Monopol und die Exklusivität der Hochkultur stark relativiert hat. Für die theoretische Konzeptionierung einer Generationen-Theorie hat das zweierlei Konsequenzen: Zum einen haben sich die sozialen Gruppen, die in der vorangeschrittenen Moderne generationsfähig sind, stark über die ursprünglichen Eliten hinaus erweitert. Zum anderen dient nicht mehr allein die Hochkultur als Kristallisationskern einer Bildung von historischen (Elite-) Generationen, sondern wir müssen auch die Popularkultur (beispielsweise die modernen Massenmedien) als Kraft in Rechnung stellen, die zum Generator von Generationenbildung werden kann.

Eine Reformulierung der Mannheimschen Theorie in dem Sinne, wie sie hier angedeutet wurde, würde die Möglichkeiten dieses Beitrages übersteigen. Ich möchte daher im weiteren Verlauf des Beitrages lediglich versuchen, einige empirische Beispiele und theoretische Hinweise zu geben, um die angedeutete Richtung einer erweiterten und reformulierten Generationskonzeption zu plausibilisieren.

\section{Generationen und das Problem der gesellschaftlichen Integration in der Moderne}

Sehen wir historische (Jugend)Generationen heute in erster Linie noch als dynamische Faktoren im gesellschaftlichen, insbesondere kulturellen Wandel, wie Mannheim dieses tat? Oder interessiert uns mittlerweile mehr die integrierende Funktion von Generationen, die diesen in einer enttraditionalisierten Moderne zufallen könnte: symbolische Heimat für individualisierte Einzelne zu sein, die ihre angestammten sozialen Orte - Stand, Klasse, Nation, stabile Groß-Milieus, Nachbarschaft - verloren haben? Es finden sich genügend Fingerzeige in der aktuellen Forschungslandschaft, die auf eine solche Umwertung hinweisen. Wenn dem so ist, dann sollte das Deutungsmuster Generation um das Deutungsmuster Milieu ergänzt werden. Im Mikro- und Mesobereich der sozialen Welt finden Prozesse der sozialen Einbettung statt, die zugleich auch einer generationellen Ordnung unterliegen. Eine der Fragen in diesem wenig bearbeiteten Feld wäre dann: Lösen im 21. Jahrhundert milieuspezifisch begrenzte Generationen die „Hochform“ gesamtgesellschaftlicher Generationen ab? Daran könnte sich die Frage anschließen, ob im Diskurs um historische Generationen nicht immer schon Fragen von Milieus versteckt waren. Das gilt insbesondere für den $\mathrm{Be}$ zug auf generationelle Kerngruppen (Generationseinheiten), die bei einer milieuspezifischen Analyse als Gruppen kultureller Elite-Milieus zu identifizieren wären.

\section{Ein sozialer Ort für die individualisierten Einzelnen?}

Gesamtgesellschaftlich wirkende Generationen eignen sich nur bedingt dazu, die sozial ortlos gewordenen modernen Subjekte sozial wieder "einzubetten“. Das wird sichtbar, wenn ein Generationenforscher wie Heinz Bude die identitätsver- 
leihende Kraft einer außergewöhnlich gut sichtbaren Generation wie die 68er für die individuellen Teilhaber an dieser Generation untersucht (Bude 1995; vgl. Bude 2000, S. 19). Sein skeptisches Resümee über die "biographische Relevanz" einer „Selbstidentifikation“ mit einer solchen Generation sei hier kurz angeführt (Bude 2000, S. 25):

"Die Formel von der Achtundsechziger-Generation stellt eine soziale Konstruktion mit vagem Ereignisbezug und geringer Beteiligungsverpflichtung dar, die dem einzelnen eine Orientierung im Fluß der Geschichte ermöglicht ... So gesehen stiften generationelle Selbstidentifikationen keine dauernde, sondern nur eine gelegentliche Orientierung. Das Mitschwingen dient mehr der momentanen Lebensgefühlsversicherung als der generellen Handlungsstrukturierung." (ebd., S. 33)

Nun teilen nur wenige Menschen das Glück oder Schicksal, einer identitätsstiftenden "Jahrhundertgeneration" wie den 68ern anzugehören. Wie ist es dann mit all jenen Jahrgängen bestellt, die dazwischenfallen, die einer jener "stillen“, nschweigenden ${ }^{*}$, ${ }_{n}$ stummen " Generationen angehören, deren Generation zu den "Zaungästen“ der Zeitgeschichte erklärt wird, deren Jahrgänge zu den „Nachzüglern“ rechnen - allesamt mit einer Generation verbunden, die zu keinem tragfähigen "Zusammenhang“, zu keiner öffentlich profilierten "Einheit“ zusammenfand?

Fragen wir mit den Augen Mannheims zurück. Das Deutungsmuster Generation als eine Kategorie sozialer Einbettung für die vereinzelten Einzelnen, nachdem „Nation“ oder „Klasse“ nicht mehr zur Verfügung stehen - dies war gewiß kein Problem, das Mannheim in den 1920er Jahren sonderlich bewegte. Er wollte mit dem Konzept der historischen Generation einen Wirkfaktor identifizieren, der - neben anderen Faktoren - Wandlungsprozesse in der Geschichte deutbar machte. Die Debatten, die gegen Ende des 20. Jahrhunderts um die Auflösung von soziokulturellen und soziomoralischen Milieus geführt werden (Mooser 1983; Hradil 1987), hätten ihn angesichts der politischen Durchschlagskraft von Arbeiter-, katholischen oder konservativen Milieus in der Weimarer Epoche wahrscheinlich in Erstaunen versetzt. Auch die soziologische „Individualisierungsdebatte ${ }^{\star}$ der 1980er Jahre lag für Mannheim in den 1920er Jahren noch in einer deutlichen historischen Ferne.

\section{Generationen und Milieus}

Wenn es um die soziale Verortung von Individuen in der Moderne geht, wird in den Sozialwissenschaften gewöhnlich ein anderes Deutungsmuster bemüht, das des „Milieus“. Milieus haben den Vorzug einer vergleichsweise eindeutigen Ortsund Gruppenbestimmung (vgl. Matthiesen 1998). In ihnen halten sich Menschen alltäglich und über längere Strecken ihrer Lebensgeschichte auf; hier sind sie als partizipierende Akteure gefragt, und hier sind vielfältige Anknüpfungspunkte für eine soziale und kulturelle Verortung der Einzelnen gegeben. Ein Milieu vermag ebenso als "konjunktiver Erfahrungsraum“ (Bohnsack 1998, S. 119) im Sinne von 
Mannheim zu fungieren wie ein Generationszusammenhang. Das Deutungsmuster Milieu suggeriert allerdings traditionell eine gewisse Zeitlosigkeit, einen Stillstand in der Zeit. Es ist vielleicht kein Zufall, daß selbst historische Untersuchungen zu Milieus oftmals als Querschnitte angelegt sind (in der Art: das katholische Milieu im Wilhelminischen Kaiserreich oder in der Weimarer Republik) und daß erst in jüngster Zeit historische Längsschnitte und Entwicklungsprozesse von Milieus in den Blick genommen werden (Köster; Liedhegener 1998). Milieus kennen ihrerseits oftmals keine Generationen - jedenfalls in der wissenschaftlichen Rekonstruktion. Verknüpfen wir das Deutungsmuster Generation aber mit der Milieuanalyse, so stellen wir rasch fest, daß die Geschlossenheit der Milieus sich unter dem Druck der dort befindlichen Generationen auflöst und die Dynamik von Wandlungsprozessen erkennbar wird. Jedes Milieu bildet ein eigenes System von Generationsbeziehungen aus, autopoietisch gleichsam, das die am Milieu beteiligten Akteure einbindet. Das milieugebundene generationelle System kann sich erheblich vom System der gesamtgesellschaftlichen Generationen und deren Jahrgangs- und Ereignisdatierungen unterscheiden. Meines Erachtens öffnen sich aufschlußreiche neuartige Forschungsfelder, wenn wir die Deutungsmuster Generation und Milieu konsequenter und häufiger aufeinander beziehen (z. B. Steinrücke 1986 für den Fall betriebsbezogener Milieus und Generationen). Wie gestalten sich beispielsweise Altersverläufe und Generationsabfolgen im Milieu des Leistungssports? Wie stellen sich Generationen im Milieu von Zuwanderungsgruppen dar? Wie im Milieu von Stadtteilen, Jugendverbänden oder Pflegeinstitutionen des Alters?

Mannheim läßt in seinem Modell der Generationen nur an einer Stelle ausdrücklich einen Milieubezug zu - nämlich immer dann, wenn es sich um ein generierendes Milieu handelt, aus dem eine profilierte Generationseinheit hervorgeht. Nur in dieser Phase der historischen Emergenz denkt er sich „eine konkrete Gruppe" (Mannheim 1964, S. 548), die in besonderer Weise dazu prädestiniert sei, „jener neuartigen ,Erlebnis-Schichtung“ Ausdruck zu verleihen“, so daß sie - wie eine zündende Idee - auf andere Gruppen in gleicher oder benachbarter Generationslagerung überspringe. Heinz Bude demonstriert eindrucksvoll am Beispiel der 68er Generation, wie es zweier Jahrzehnte, der 1970er und der 1980er Jahre, bedurfte, um eine kleine sozial vernetzte studentische Bewegung, die auf einzelne Universitäten und Fachkulturen begrenzt war, Schritt für Schritt in eine Generation der "Achtundsechziger" umzutaufen (Bude 2000, S. 25), mit der sich zunehmend mehr Angehörige verwandter Jahrgänge und Lebenslagen identifizierten.

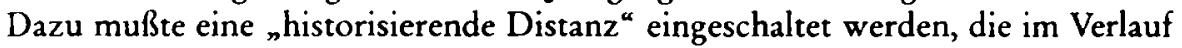
der 80er Jahre und danach zunehmend anwuchs.

„Die Achtundsechziger-Generation erfuhr in den 80er Jahre eine erhebliche retrospektive Vermehrung. Je mehr die Jahrgangsgemeinschaft zum definierenden Merkmal erhoben wurde, um so mehr Angehörige meldeten sich. ${ }^{\text { }}$ - Je mehr Zeit vergeht, um so deutlicher treten gemeinsame Stimmungen, Grundgefühle und Probleme hinter verschiedenen politischen und ideologischen Orientierungen hervor." (Bude 2000, S. 26, 27 f.) 


\section{Gibt es historische Hochphasen der Generationenbildung?}

Den historischen Begriffen des soziokulturellen bzw. soziomoralischen Milieus auf nationaler Ebene - wie beispielsweise dem politischen Katholizismus oder der Arbeiterbewegung zwischen 1850 und 1950 - sind von der Geschichtswissenschaft definierte, langfristige Epochen und Perioden zugewiesen worden. Die forschenden Historiker sprechen ihnen eine Entstehungsphase, eine Hochphase und eine Verfallszeit im Kontext eines Jahrhunderts zu. Als Schlüssel- oder Ankertext gilt in diesem Fall die frühe Arbeit von Rainer M. Lepsius (1966). Können wir in ähnlicher Weise von einem historischen Anfang und von einem ebensolchen historischen Ende der gesellschaftlich begünstigten Herausbildung von Generationen sprechen?

Am Ende des 20. Jahrhunderts wurde von Seiten postmodernen Denkens die Frage aufgeworfen bzw. es wurde proklamiert, daß die historische Hochform von gesellschaftlichen Generationen nun zu einem geschichtlichen Ende gekommen sei. Ein solches Ende wurde seit den 1980er Jahren ja auch für andere Institutionen der Moderne - für Kindheit, Jugend, soziale Klassen, lokale Milieus usw. - bereits behauptet. Insofern verwundert diese Erklärung von Endzeit nicht mehr. Im Fall der gesamtgesellschaftlich verankerten Generationen wurde für ein wahrscheinliches Ende vor allem der Verfall einer bürgerlichen Öffentlichkeit im Kontext der Entwicklung einer individualisierenden kommerziellen Medienkultur geltend gemacht. Generationen setzen entsprechend dieser emphatischen Auffassung eine gesamtgesellschaftlich wirksame Öffentlichkeit voraus. Als eine solche letzte Hochform wurde die 68er-Generation ausgerufen (vgl. Hörisch 1997a). Eine Analyse der historischen Bedingungen, die zu bestimmten epochalen Generations-Gestalten führen können, wäre gewiß lohnenswert, steht aber noch aus.

\section{Jugendgenerationen. Vom „Mythos Jugend" zur Entzauberung der Jugend}

Mit diesen beiden Etiketten, Mythos und Entzauberung, könnte man die historische Laufbahn der Altersphase Jugend zwischen Beginn und Ende des 20. Jahrhunderts umschreiben. Mannheim argumentierte noch vor dem Hintergrund eines hochstilisierten Jugendbegriffs. Ein Dreivierteljahrhundert später ist der "Mythos Jugend" arg verblaßt. Historische Jugendforscher attestieren der heutigen Jugend, daß sie keinen sicheren Platz mehr im Gefüge der Altersgruppen einnehme und in der jüngsten Geschichte noch nach ciner sinnvollen und akzeptierbaren Aufgabenbestimmung in Frieden wie im Krieg suche (vgl. Gillis 1993; Zinnecker 2000a; 2000b). Demographisch befindet sich die Jugend auf dem Weg zu einer Bevölkerungs-Minderheit, die im Rahmen von alternden Wahl-Demokratien politisch durch ältere Altersgruppen majorisiert zu werden droht.

Angesichts der Entzauberung von Jugend nimmt es nicht wunder, daß sich die Stimmen mehren, die eine Jugendgenerationstheorie wenig attraktiv finden. Werden, so wird beispielsweise eingewandt, entscheidende Lebenserfahrungen nicht lebenslang, sozusagen gleichberechtigt in allen Lebensabschnitten gemacht? So wagt sich etwa die Biographieforscherin Gabriele Rosenthal weit vor, wenn sie erklärt: 
„Die Lebensphase, in der für die Bildung eines Generationszusammenhangs konstitutive Erfahrungen gemacht werden, kann von der frühen Kindheit bis ins spätere Erwachsenenalter reichen." (Rosenthal 2000, S. 165)

Solche Auffassungen sind eine Art Frontalangriff auf das kunstvolle Analysegebilde, das Mannheim uns als Erbe hinterlassen hat. Aber ist diese Konfrontation auch sachlich berechtigt?

Der Besuch einiger Suchmaschinen des Internets belehrt uns über eine massive semantische Verschiebung. Der Suchbefehl „Generation“ (deutsch) deckt eine erfreuliche Popularität des Begriffs auf - er generiert Hunderttausende von Verweisen. Die Durchsicht des auf diese Weise erzeugten Sprachmaterials, das überwiegend den Domänen Alltag, Ökonomie und Medien entnommen ist, deckt eine semantische Strukturverschiebung auf, die im Wissenschaftsdiskurs noch kein entsprechendes Echo gefunden hat. Das Begriffsfeld Generation hat sich mittlerweile von den lebenden, menschlichen Generationen auf die technischen, medialen Generationen bin ausgedehnt bzw. verschoben. Die "Generationen" der technischen Geräte und medialen Angebote erscheinen zahlenmäßig viel präsenter, und sie spiegeln auch eine größere Vielfalt als die Angebote der lebenden Human-Generationen. Die überzeugendsten Generationen sind heute offenkundig die Generationen technologischer, medialer Neuerungen, die in hoher Geschwindigkeit und - anders als die humanen Neuankömmlinge, die in relativ kontinuierlicher zeitlicher Abfolge geboren werden - im Gleichschritt die humane Welt erobern. Diese technologischen Generationen sind noch - anders als die humane jüngere Generation - wirkliche Hoffnungsträger: Wir erwarten von ihnen Fortschritt. "Die nächste Generation kommt ${ }^{*}$, das ist ein starkes Werbeargument geworden. Die Verbesserungen sind absehbar. "The next generation " besitzt mehr Power, mehr Komfort, mehr Sicherheit, mehr Kompetenz (vgl. hierzu auch Sackmann 1992, S. 210). Sprichwörtlich geworden sind mittlerweile die „Chip-Generationen " der PCs, die der Literatur- und Medienforscher Jochen Hörisch in seiner Auseinandersetzung mit dem sozialwissenschaftlichen Konzept der Generationen nicht ohne Genugtuung gegen die humanen Generationen ausspielt: „88-, 286-, 386-, 486- und Pentium-Prozessoren und nicht $68 \mathrm{er}$ oder $89 \mathrm{er}$ bestimmen die Lage und die Lager" (Hörisch 1997, S. 14 f.).

Angesichts dieser paradoxen Umkehrung nimmt es nicht wunder, wenn die jüngsten humanen Generationen sich an dieses Werbemedium anhängen und es zur Konstruktion einer generationalen Lage nutzen. So benennt ein jüngerer Autor, Florian Illies (2000), seine eigene Generation in einem vielgelesenen Essay nach einem bekannten Automodell „Generation Golf“ . In dieser kollektiven WirAutobiografie wird die Rückübertragung ironisch und literarisch nachvollzogen. Die eigene Generation wird vom Autor nach dem populären Fahrzeug und den dazugehörenden Werbekampagnen modelliert. Die Selbstidentifikation mit seiner Generation wird zur Identifikation mit der Automarke. Bereits im Buch-Titel wird die "Generation Golf“ einer „Inspektion" unterzogen. Es handelt sich um "die zwischen den Baujahren 1965 und 1975 Geborenen“. Am Ende faßt der Autor selbstironisch das Motto seiner „Generation Golf* mittels eines der seiner- 
zeit bekannten Golf-Werbeslogans zusammen, die die einzelnen Kapitel seines und seiner Generation Lebenslauf leitmotivisch begleiten: „Die Suche nach dem Ziel hat sich somit erledigt. Es gibt kaum einen Satz, der die Lebensphilosophie unserer Generation präziser auf den Punkt bringt“ (Illies 2000, S. 189).

\section{Mögliche Träger generationeller Erfahrung: \\ Welche Bevölkerung und welche Kultur?}

Mannheim geht, das wurde oben bereits hervorgehoben, wie die anderen Generationstheoretiker im ersten Drittel des 20. Jahrhunderts stillschweigend von einem Elitemodell aus. Generationseinheiten werden aus miteinander konkurrierenden kulturellen und politischen Eliten der jüngeren Generation konstituiert. Sind es in den älteren Theorien zunächst literarisch, künstlerisch, musikalisch tätige Gruppen, erweitert er diese Eliten um politische Gruppen. Auch hier hat sich mittlerweile eine gravierende Änderung vollzogen, die das Modell der Generationen zu berücksichtigen hat.

\section{Der Umkreis potentieller Trägergruppen bat sich erweitert}

Die Erweiterung des potentiellen Personenkreises, der „generationenfähig “ im emphatischen Sinn wird, geht Hand in Hand mit der Ausweitung der kulturellen Gebiete, die zum Kristallisationspunkt generationeller Erfahrung werden können. Zur Hochkultur gesellten sich Popularkultur, Alltagskultur, Medienkultur u.ä. Zum zweiten läßt sich ein Zusammenhang mit der Inklusion wachsender Gruppen in die biographische und generationelle Öffentlichkeit herstellen. Erinnerungen veralltäglichen sich, verkindlichen sich, vervielfältigen sich. Die Erweiterung der beteiligten Gruppen läßt sich gut an der Popularisierung von Erinnerungen an prägende Generationenerfahrungen im 20. Jahrhundert ablesen. Erinnert sei an die historischen Wellen, die zur Arbeiter-Autobiographie, zur FrauenAutobiographie oder zur ländlich-bäuerlichen Autobiographik führten. Mit der mündlich überlieferten Geschichtsschreibung erhielten auch nicht-literaturfähige oder zuvor nicht öffentlichkeitsfähige Gruppen die Möglichkeit, generationale Erfahrungen zu veröffentlichen und zur Identitätsfindung zu nutzen. Die Geschichtsschreibung von prominenten Jugend-Generationen ist traditionell bürgerlich und männlich orientiert. Das wurde und wird mittlerweile in der Forschungsliteratur nachhaltig korrigiert.

Ein anderer Nutznießer des historischen Verfalls des Mythos Jugend ist die Lebensphase Kindheit geworden. Sic wird Ende des 20. Jahrhunderts zunehmend als prägende kulturelle Lebensphase gedeutet. Die frühe generelle Beteiligung der jüngeren Generation an Medien- und Warenkonsum schafft kollektive Identitäten, die sich zu Generationsgestalten verdichten können. Träger einer solchen generationellen Erinnerungstätigkeit über Kindheit sind maßgeblich bereits junge Erwachsene und nicht mehr, wie historisch überliefert, Menschen zu Beginn ihres Ruhestandes. 


\section{Kindergenerationen - die früben prägenden Konsum- und Medienjabre}

„Playmobil ist sicherlich das Prägendste, was unserer Generation passiert ist“ (Illies 2000, S. 19). Mit dieser Formel weist Illies (Jahrgang 1971) auf die folgenreiche Verschiebung der prägenden Jahre von der Jugend- auf die Kindheitsphase hin. Die Jugendphase droht durch Kindheit in ihrer biographischen Bedeutung für eine Bildung von generationellem Bewußtsein entthront zu werden. Möglich wird das durch einen Themenwechsel. Was eine junge Generation prägt, das sind nicht mehr notwendigerweise historisch-politische Großereignisse, sondern die kleinen Dinge des Konsumentenalltags. In dem Maß, in dem auch Kinder in die Welt des Massenkonsums integriert werden, was in den Jahrzehnten nach dem $Z$ weiten Weltkrieg einsetzt, historisiert sich Kindheit potentiell. Kindergenerationen lassen sich danach unterscheiden, durch welche Konsumerlebnisse sie in Vor- und Grundschuljahren geprägt worden sind.

Ein beliebiges Beispiel unter vielen mag den Sachverhalt erläutern. Susanne Pauser cröffnete im November 1997 - sie war zu der Zeit dreißig Jahre alt - im österreichischen Internet-Forum blackbox.net "für meine Generation eine OnlineNewsgroup“ (Pauser, Ritschel 2000, S. 10). Der Ausschreibungstext lautete, in Auszügen:

"Liebe Kinder der siebziger Jahre!

Bist Du um die 30 und kannst Du Dich noch an Dinge erinnern, die 20 oder mehr Jahre zurückliegen? Dann sei herzlich willkommen in dieser neuen Online-Konferenz. Und das ist das Thema:

Bin ich die einzige, die sich an eine klebrige Süßigkeit namens Leckerschmecker erinnert? Hat außer mir noch jemand alle verfügbaren Groscherl in Sugus und Stollwerk umgesetzt? Kann jemand noch den Faserschmeichler-Song singen? ...

Erzähit mir von Euren Erinnerungen an unsere Kindheit in den Siebzigern, von Produkten, TV-Sendungen, Werbespots und Lebensmitteln, die Ihr gekannt habt und die es vielleicht nicht mehr gibt.

Ich freue mich auf Eure Geschichten!

Fure Susanne"

Eine Einladung einer jungen Erwachsenen also zu einer erinnernden Erzählrunde an die mittlerweile zwei Jahrzehnte zurückliegende Konsum-, Werbe- und Medienkindheit, wobei besonderes Gewicht auf die sinnliche Erfahrung von Süßigkeiten und anderen Lebensmitteln für den Kindermarkt gelegt wird! Das Internet-Forum wurde ein großer Erfolg, Tausende von erwachsen gewordenen Kindern der 1970er Jahre beteiligten sich.

Junge Erwachsene stiften über das Internet eine Generationseinheit, wobei der Zusammenhang nicht durch die Jugendphase hervorgebracht wird, sondern durch die Gemeinsamkeiten einer kommerziellen Kinderwelt, eines medial (mit)geteilten Massenkonsums. Diese Verjüngung der Herstellung von generationellem Bewußtsein sollte beachtet werden, die mit einer generellen Tendenz zur Verjüngung biographischer Erinnerung und Erzählung einhergeht. ${ }^{15}$ Die hier beobachtbare

15 Als ein empirischer Indikator sei angeführt: Das Alter, in dem Autobiographien erstmals auf dem Buchmarkt veröffentlicht werden, sank im Verlauf des 20. Jahrhunderts deutlich. 
Historisierung von Kindheit, die unterscheidbare Kindheits-Generationen hervorbringt, wurde seit Anfang der 1980er Jahre auch wissenschaftlich geadelt, als sich in Deutschland eine pädagogisch-sozialwissenschaftliche Kindheitsforschung aufmachte, die Geschichte des 20. Jahrhunderts nach unterscheidbaren historischen Kindheitsgenerationen zu mustern. Eines der vielzitierten Ergebnisse, in dem Kindergenerationen nach 1945 identifiziert werden, trägt beispielsweise den bezeichnenden Titel ${ }_{n}$ Kriegskinder. Konsumkinder. Krisenkinder“ (Preuß-Lausitz et. al. 1983).

\section{Die kulturellen Generatoren von Generationserfabrungen haben sich vermehrt}

Im Geist der klassischen Generationstheorien konzentrierte sich Mannheim auf Phänomene der Hochkultur und der offiziellen politischen Kultur. Im Vergleich zum ersten Viertel des Jahrhunderts treffen wir heute auf eine Vielzahl von weiteren möglichen generationsbildenden kulturellen Institutionen. Folgende Attribuierungen von Generationen aus dem Ende des Jahrhunderts vermögen die Erweiterung, die mittlerweile stattgefunden hat, anzudeuten: "Technikgenerationen (Weymann 2000) - „Mediengenerationen" (Hörisch 1997a) - „Wohlfahrtsgenerationen " (Leisering 2000) - "Konsumgenerationen“ (Kindheitsforschung) usw. Etwas systematischer können wir davon sprechen, daß sich im Gang durch das Jahrhundert der ( „bürgerliche“) Kulturbegriff, der streng normativ und hochkulturell konnotiert war, deutlich erweitert hat. Wir beziehen heute Popular- und Alltagskultur mit ein. Die Kulturanthropologie, später die inländischen cultural studies, haben uns nahegebracht, jeder Form menschlicher Praxis den Rang von Kultur zuzuerkennen. Auch technische Bereiche werden nicht mehr als Zivilisation ausgegrenzt, wie es deutscher Semantik entsprach, sondern als Kultur einbezogen. Damit sind weite Kreise der Bevölkerung in Kultur eingebunden und können daraus prägende Erfahrungen gewinnen. Das gilt auf der einen Seite für die Standard-Massenkultur (Konsum und Medien), die erst seit dem Zweiten Weltkrieg für die Mehrheit der Bevölkerung zugänglich wurde. Das gilt auch für den neuartigen Bereich der Szenen und Subkulturen, die generationsprägende Bedeutung erhalten können. Das Deutungsmuster Generation hat einen offeneren Blick für die Art und Weise der kulturellen Prägungen von Generationen entwickelt.

\section{Mediengenerationen}

Neben Verweisen auf Konsum und Alltagstechnik (Weymann 2000) werden jüngere Generationen zunehmend mit Medienetiketten tituliert. So finden wir Generationslabels wie „Wir Fernsehkinder" (Wüllenweber 1994), „Die Windows-Generation“ (Schwab, Stegmann 1998) oder „Generation @“ (Opaschowski 1999). Solche Etikettierungen sind nicht nur als billige Diskursmünzen zu belächeln, sie zeigen auch eine beachtenswerte Tendenz an. Ende des 20. Jahrhunderts treten Medien als prägende Kindheits- und Jugenderfahrungen hervor. Mit den Labels 
werden jeweils Leitmedien eines Zeitabschnitts herausgestellt. „Fernseh-Generation" bezieht sich auf die Zeiten des öffentlich-rechtlichen Fernsehens, vor der Öffnung des privaten und Werbefernsehens um 1985. „PC-Kids“ oder „Windows-Generation“ verweist auf die Phase der Dominanz häuslicher „Personal Computers“, „Generation@“ wiederum thematisiert die historische Phase der elektronischen Vernetzung dieser digitalisierten Welt. Mittlerweile hat sich ein Konsens herausgebildet, wonach die Zeit des öffentlich-rechtlichen Fernsehens als Trennlinie zwischen den Mediengenerationen geeignet erscheint. Das kollektive Fernseherleben einer ganzen Generation konstituierte danach eine letzte gesamtgesellschaftliche Mediengeneration, bevor die Individualisierung des $\mathrm{Me}$ diengebrauchs dem ein Ende setzte. In den Worten von Thomas Maurer (2000, S. 204): „Kollektive kindliche Fernseherlebnisse werden für immer ein Privileg unserer Generation bleiben." Aus der Perspektive von Medien- und Kommunikationswissenschaftlern bilden die unterschiedlichen Medienerfahrungen sogar die entscheidende Differenz ab, die die 68er und die 89er Generationen trennt. Während die 68er noch in einer halbwegs intakten "Gutenberg-Galaxis“, gekoppelt allerdings mit der öffentlich-rechtlichen Fernsehwelt lebten, lebten die $89 \mathrm{er}$ dagegen bereits in einer elaborierten Medienwelt am Ende jener Galaxis (vgl. Hörisch 1997; Steiner 1997).

\section{Abschließende Einschätzungen}

Eine bestimmte Stoßrichtung des Beitrages sollte deutlich geworden sein: Die konzeptionelle Fassung, die Mannheim der Analyse von Generationen $1928 \mathrm{gab}$, hat sich als außerordentlich anregend erwiesen, und dieser Anregungsgehalt ist längst noch nicht ausgeschöpft. Auf der anderen Seite transportiert das Konzept auch einige gewichtige historische Engführungen aus der Entstehungszeit mit sich. Diese könnten sich künftig als gedankliche Barriere für eine adäquate Untersuchung der generationellen Ordnungen und Verflechtungen dieses Jahrhunderts erweisen. Auf eine problematische Einengung stießen wir im Verlauf des Beitrags wiederholt: Mannheims Konzept zielt ausdrücklich nur auf eine makrosoziologische Theorie der Generationen - und hier wiederum nur auf den Typus der politisch-gesellschaftlichen Generation. Zusätzlich wurde das Konzept der Generation von ihm mehr oder weniger eng an die Thematik jugendlicher Generationen gebunden.

Als generelle Strategie für die Entwicklung einer künftigen Theorie-Architektur bietet sich an, die Begrifflichkeit der Generationen und die Analyse von Generationen-Dynamik konsequent auszudifferenzieren. Die Abfolge von und das Wechselspiel zwischen Generationen sollte als ein Thema fokussiert werden, das auf allen Ebenen des sozialen Lebens, auf der Makro- ebenso wie auf der Mesound Mikroebene, und in allen institutionellen Handlungsfeldern eine bedeutsame Rolle spielt. Im Gegenzug ist daran zu denken, neben regionalen und nationalen 
auch internationale und globale Bildungen von Generationen zu analysieren. Die mehr oder minder stillschweigende Bindung des wissenschaftlichen Generationen-Diskurses an kulturelle Eliten und an Bereiche der Hochkultur sollte aufgegeben werden. Statt dessen wären alle generationsfähigen Bevölkerungsgruppen und alle kulturellen Bereiche, auch die der Massen- und Medienkultur, die zu Kristallisationskernen von Generationen werden können, zur Analyse zuzulassen. Für Zwecke einer Historisierung der Generationen-Analyse wäre daran zu denken, solche Epochen und Kulturen zu identifizieren, die in besonderer Weise die Emergenz von Generationen fördern (Hoch-Zeiten der Generationsbildung) und, auf der Gegenseite, sozial- und kulturgeschichtliche Bedingungen zu formulieren, die der Entstehung von Generationen eher hinderlich sind. Vieles spricht dafür, künftig das Konzept der Generationen-Analyse, das Karl Mannheim entwickelt hat, nicht lediglich unbefragt anzuwenden, zu tradieren oder zur Legitimierung eigener Studien zu benutzen. Sinnvoller wäre es, die Empirie wo immer möglich mit begrifflichen Anstrengungen zur Adaptation des Generationen-Konzeptes an gegenwärtige Fragestellungen und Diskurse zu verbinden.

\section{Literatur}

Bilstein, Johannes (1996), Zur Metaphorik des Generationenverhältnisses, in: Liebau, E., Wulf, $C h$. (Hrsg.), Generation. Versuche über eine pädagogisch-anthropologische Grundbedingung (Weinheim) 157-189

Bohnsack, Ralf (1998), Milieu als konjunktiver Erfahrungsraum. Eine dynamische Konzeption von Milieu in empirischer Analyse, in: Matthiesen, $U$. (Hrsg.), Die Räume des Milieus (Berlin) 119-132

Breitsamer, Joachim (1976), Ein Versuch zum „Problem der Generationen“, in: KZfSS 28,3, $451-478$

Buchbofer, Bernd, Friedrichs, Jürgen, Lüdtke, Hartmut (1970), Alter, Generationsdynamik und soziale Differenzierung. Zur Revision des Generationsbegriffs als analytisches Konzept, in: KZfSS 22, 2, 300-334

Bude, Heinz (1995), Das Altern einer Generation. Die Jahrgänge 1938 bis 1948 (Frankfurt a.M.)

Bude, Heinz (1997), Die Wir-Ebene von Generationen, in: Berliner Journal für Soziologie 7 H. 2. (Themenheft N. Elias)

Bude, Heinz (2000), Die biographische Relevanz der Generation, in: Kohli, M., Szydlik, $M$. (Hrsg.), Generationen in Familie und Gesellschaft (Opladen) 19-35

Demartini, J. $R$. (1985), Change agents and generational relationships: A revaluation of Mannheim's problems of generations, in: Social Forces $64,1 \mathrm{ff}$.

Dilthey, Wilhelm (1875/1924), Über das Studium der Geschichte der Wissenschaften vom Menschen, der Gesellschaft und dem Staat, in: Diltbey, W., Gesammelte Schriften, Bd. V, hrsg. v. Misch, G. (Leipzig, Berlin)

Dilthey, Wilhelm (1870/1922), Leben Schleiermachers, Bd. I (Berlin, Leipzig)

Dilthey, Wilhelm (1927/2001), Der Aufbau der geschichtlichen Welt in den Geisteswissenschaften, in: Dilthey, W., Gesammelte Schriften, Bd. VII, hrsg. v. Groethuysen, B. (Leipzig, Berlin) 79-188 (zitiert nach der Ausgabe von 2001, hrsg. v. Riedel, M. [Frankfurt a.M.]; die Erstausgabe erschien 1910 als Abhandlung der Berliner Akademie der Wissenschaften)

Friedeburg, Ludwig von (Hrsg.) (1965), Jugend in der modernen Gesellschaft (Köln, Berlin) 
Friedrich, Walter (1990), Bemerkungen zum Generationskonzept, in: Deutsche Zeitschrift für Philosophie 38,1, 42-50

Gillis, John R. (1993), Vanishing Youth: The Uncertain Place of the Young in a Global Age, in: Young 1 Nr. 1

Heidegger, Martin (1927/1953), Sein und Zeit (Tübingen; zitiert nach der 7. unveränderten Auflage 1953; ursprünglich in: Jahrbuch für Phänomenologie und phänomenologische Forschung, hrsg. v. Husserl, E., Bd. VIII)

Hermann, Ulrich (1987), Das Konzept der „Generation“. Ein Forschungs- und Erklärungsansatz für die Erziehungs- und Bildungssoziologie und die Historische Sozialisationsforschung, in: Neue Sammlung 27,3, 364-377 (Wiederabdruck in: Hermann, U., Historische Bildungsforschung und Sozialgeschichte der Bildung [Weinheim 1991] 319-330)

Hradil, Stefan (1987), Sozialstrukturanalyse in einer fortgeschrittenen Gesellschaft. Von Klassen und Schichten zu Lagen und Milieus (Opladen)

Hörisch, Jochen (Hrsg.) (1997a), Mediengenerationen (Frankfurt a.M.)

Hörisch, Jochen (1997b), Was generiert Generationen: Literatur oder Medien? Zur Querelle allemande zwischen Achtundsechzigern und Neunundachtzigern, in: Hörisch, J. (Hrsg.), Mediengenerationen (Frankfurt a.M.) 7-15

Illies, Florian (2000), Generation Golf. Eine Inspektion (Berlin ${ }^{4} 2000$ )

Käsler, Dirk (1984), Die frühe deutsche Soziologie 1909 bis 1934 und ihre EntstehungsMilieus. Eine wissenschaftssoziologische Untersuchung (Opladen)

Koebner, Thomas, Janz, Rolf-Peter, Trommler, Frank (Hrsg.) (1985), „Mit uns zieht die neue Zeit". Der Mythos Jugend (Frankfurt a.M.)

Köster, Christoph, Liedhegener, Antonius (1998), Historische Milieus als Forschungsaufgabe. Zwischenbilanz und Perspektiven (Tagungsbericht), in: Westfälische Forschungen 48, 593-601

Kohli, Martin (Hrsg.) (1978), Soziologie des Lebenslaufs (Darmstadt; Neuwied)

Kreutz, Henrik (1983), Generationsbildung als eine Form der Vergesellschaftung in der Situation sozialen Wandels. Mannheims Theoric und ihre Fortführung in der Forschung der Gegenwart, in: Angewandte Sozialforschung 11,4/5, 385-394

Lange, Andreas (1999), "Generationenrhetorik" und mehr. Versuche über ein Schlüsselkonzept, in: Sozialwissenschaftliche Literatur Rundschau 22, 39, 71-86

Leisering, Lutz (2000), Wohlfahrtsstaatliche Generationen, in: Kobli; M., Szydlik, M. (Hrsg.), Generationen in Familie und Gesellschaft (Opladen) 59-76

Lepsins, M. Rainer (1966), Parteiensystem und Sozialstruktur. Zum Problem der Demokratisienung der deutschen Gesellschaft, in: Abel, Wilhelm (Hrsg.), Wirtschaft, Geschichte und Wirtschaftsgeschichte (Stuttgart) 371-393 (Wiederabgedruckt in Ritter, Gerhard A. [Hrsg.], Deutsche Parteien vor 1918 [Köln 1973] 56-80)

Lukács, Georg (1923), Geschichte und Klassenbewusstsein (Berlin)

Mannheim, Karl (1928), Das Problem der Generationen, in: KVJS (Kölner Vierteljahresschrift für Soziologie) 7, 157-185 u. 309-330 (Wiederabdruck in Wissenssoziologie [1964] 509-565)

Mannheim, Karl (1941), Das Problem der Jugend in der modernen Gesellschaft (Wiederabdruck in: Mannbeim, K., Diagnose unserer Zeit [Zürich 1951])

Mannheim, Karl (1964), Wissenssoziologie. Auswahl aus dem Werk, hrsg. v. Wolff, K. $H$., (Berlin, Neuwied)

Mannheim, Karl (1980), Strukturen des Denkens, hrsg. v. Kettler, D., Meja, V., Stehr, N., (Frankfurt a.M.)

Mannheim, Karl (1984), Konservativismus. Ein Beitrag zur Soziologie des Wissens, hrsg. v. Kettler, D., Meja, V., Stehr, N. (Frankfurt a.M., urspr. Habilitation 1925)

Matthes, Joachim (1985), Karl Mannheims "Das Problem der Generationen“ neu gelesen. Generationen- "Gruppen“ oder "gesellschaftliche Regelung von Zeitlichkeit“ ?, in: Zeitschrift für Soziologie 14,5, 363-372

Matthiesen, Ulf (Hrsg.) (1998), Die Räume des Milieus (Berlin) 
Maurer, Thomas (2000), Biene Maja, flächendeckend, in: Pauser, S., Ritschl, W., Wickie, Slime and Paiper. Das Online-Erinnerungsalbum für die Kinder der siebziger Jahre (Reinbek b. H.) 204-208

Mentré, $F$. (1920), Les générations sociales (Paris)

Mooser, Josef (1983), Auflösung der proletarischen Milieus, in: Soziale Welt 34, 270-306

Opaschowski, Horst (1999), Generation @. Die Medienrevolution entläßt ihre Kinder: Leben im Informationszeitalter (Hamburg)

Pauser, Susanne, Ritschl, Wolfgang (2000), Wickie, Slime and Paiper. Das Online-Erinnerungsalbum für die Kinder der siebziger Jahre (Reinbek b. H., urspr. 1999)

Petersen, Julius (1926), Die Wesensbestimmung der deutschen Romantik. Eine Einführung in die moderne Literaturwissenschaft (Heidelberg, reprint: Darmstadt 1973)

Pfeil, Elisabeth (1967), Der Kohortenansatz in der Soziologie. Ein Zugang zum Generationsproblem?, in: KZfSS 19,4, 645-657

Pinder, Wilhelm (1926), Das Problem der Generation in der Kunstgeschichte Europas (Berlin, Nachdruck: München 1961)

Preuß-Lausitz, Ulf u.a. (Hrsg.) (1983), Kriegskinder. Konsumkinder. Krisenkinder. Zur Sozialisationsgeschichte seit dem Zweiten Weltkrieg (Weinheim)

Rosenthal, Gabriele (Hrsg.) (1986), Die Hitlerjugend-Generation. Biographische Thematisierung als Vergangenheitsbewältigung (Essen)

Rosenthal, Gabriele (2000), Historische und familiale Generationenabfolge, in: Kohli, M., Szydlik, M., (Hrsg.), Generationen in Familie und Gesellschaft (Opladen) 162-178

Ryder, Norman B. (1965), The Cohort as a concept in the Study of Social Change, in: American Sociological Review 30, 843-861

Sackmann, Reinhold (1992), Das Deutungsmuster "Generation“, in: Meuser, M., Sackmann, $\boldsymbol{R}$., (Hrsg.), Analyse sozialer Deutungsmuster. Beiträge zur empirischen Wissenssoziologie (Pfaffenweiler) 199-215

Schelsky, Helmut (1957), Die skeptische Generation. Eine Soziologie der deutschen Jugend (Düsseldorf, Köln)

Schwab, Jürgen, Stegmann, Michael (1998), Die Windows-Generation (München)

Simirenko, A. (1966), Mannheim's Generational Analysis and Acculturation, in: British Journal of Sociology 17, $292 \mathrm{ff}$.

Spranger, Eduard (1925), Psychologie des Jugendalters (Leipzig)

Steiner, Uwe C. (1997), „68-89*. Literarische und mediale Wendungen der Wende, in: Hörisch, J., (Hrsg.), Mediengenerationen (Frankfurt a.M.) 16-59

Steinrücke, Margareta (1986), Generationen im Betrieb. Fallstudien zur generationenspezifischen Verarbeitung betrieblicher Konflikte (Frankfurt a.M.)

Weymann, Ansgar (2000), Sozialer Wandel, Generationsverhältnisse und Technikgenerationen, in: Kohli; M., Szydlik, M., (Hrsg.), Generationen in Familie und Gesellschaft (Lebenslauf - Alter - Generationen Bd. 3, Opladen) 36-58

Wolff, Kurt H. (1978), Karl Mannheim, in: Käsler, D., (Hrsg.), Klassiker des soziologischen Denkens Bd. 2. (München) 286-387

Wüllenweber, Walter (1994), Wir Fernsehkinder. Eine Generation ohne Programm (Berlin)

Zinnecker, Jürgen (2000a), Kindheit und Jugend als pädagogische Moratorien. Zur Zivilisationsgeschichte der jüngeren Generation im 20. Jahrhundert, in: Benner, $D$., Tenorth, $H$.- E., (Hrsg.), Bildungsprozesse und Erziehungsverhältnisse im 20. Jahrhundert (ZfPäd 42. Beiheft, Weinheim, Basel) 36-68

Zinnecker, Jürgen (2000b), Jungsein in alternden Gesellschaften. Zur demographischen Ordnung der Generationen, in: Institut für soziale Arbeit (Hrsg.), Jugendförderung in Nordrhein-Westfalen (Münster) 52-72

Zinnecker, Jürgen (2002), Das Porträt. Die erste Jugendgeneration des neuen Jahrhunderts, in: Zinnecker, J., Behnken, I., Maschke, S., Stecher, L., null zoff \& voll busy (Opladen) $11-20$ 